\title{
Applications of Hückel-Su-Schrieffer-Heeger method
}

\section{Carbon-carbon bond lengths in polycyclic aromatic hydrocarbons}

\author{
Jan H. Kwapisz ${ }^{1}$ • Leszek Z. Stolarczyk ${ }^{2}$ (D) \\ Received: 4 December 2020 / Accepted: 12 April 2021 / Published online: 11 May 2021 \\ (C) The Author(s) 2021
}

\begin{abstract}
The equilibrium carbon-carbon (C-C) bond lengths in $\pi$-electron hydrocarbons are very sensitive to the electronic groundstate characteristic. In the recent two papers by Stolarczyk and Krygowski (J Phys Org Chem, 34:e4154,e4153, 2021) a simple quantum approach, the Augmented Hückel Molecular Orbital (AugHMO) model, is proposed for the qualitative, as well as quantitative, study of this phenomenon. The simplest realization of the AugHMO model is the Hückel-Su-SchriefferHeeger (HSSH) method, in which the resonance integral $\beta$ of the HMO model is a linear function the bond length. In the present paper, the HSSH method is applied in a study of $\mathrm{C}-\mathrm{C}$ bond lengths in a set of 34 selected polycyclic aromatic hydrocarbons (PAHs). This is exactly the set of molecules analyzed by Riegel and Müllen (J Phys Org Chem, 23:315, 2010) in the context of their electronic-excitation spectra. These PAHs have been obtained by chemical synthesis, but in most cases no diffraction data (by X-rays or neutrons) of sufficient quality is available to provide us with their geometry. On the other hand, these PAHs are rather big (up to 96 carbon atoms), and ab initio methods of quantum chemistry are too expensive for a reliable geometry optimization. That makes the HSSH method a very attractive alternative. Our HSSH calculations uncover a modular architecture of certain classes of PAHs. For the studied molecules (and their fragments - modules), we calculate the values of the aromaticity index HOMA.
\end{abstract}

Keywords Polycyclic aromatic hydrocarbons (PAHs) · Bond lengths · AugHMO model · HSSH method

\section{Introduction}

The world of hydrocarbon chemistry [1] is now open to materials science, where carbon-based materials offer new perspectives for electronics [2]. The $\pi$-electron hydrocarbons and all-carbon molecules (fullerenes, nanotubes, and graphene) are at the center of interest in this respect. There is a vast landscape of $\pi$-electron hydrocarbons, extending from benzene to graphene, which belongs to the polycyclic

Leszek Z. Stolarczyk

leszek@chem.uw.edu.pl

Jan H. Kwapisz

jkwapisz@fuw.edu.pl

1 Faculty of Physics, University of Warsaw, Pasteura 5, PL-02-093 Warsaw, Poland

2 Faculty of Chemistry, University of Warsaw, Pasteura 1, PL-02-093 Warsaw, Poland aromatic hydrocarbons (PAHs). This is an area for scientific prospectors equipped with proper experimental and/or theoretical tools.

Klaus Müllen and his coworkers have made seminal contributions to the chemistry, physics, and theory of PAHs; see the review article by Riegel and Müllen [3] and the references therein. Ref. [3] contains a summary of spectroscopic properties of 34 molecules belonging to various families of PAHs. These molecules have been obtained by chemical synthesis (many of them by Müllen et al.), but in most cases their equilibrium geometries are not known. The present article, based on the theory set forth in two recent papers by Stolarczyk and Krygowski [4, 5], is intended as a supplement to the Riegel and Müllen paper [3] by providing the most relevant geometry data- the carbon-carbon (C-C) bond lengths.

The equilibrium $\mathrm{C}-\mathrm{C}$ bond lengths, derived from the diffraction studies of PAHs (in the crystalline phase), vary as much as $0.15 \AA$ [6]. This variability of bond lengths is a consequence of the coupling between the nuclear framework and the system of mobile electrons that occupy the molecular orbitals of the $\pi$-symmetry (the $\pi$ MOs) [4, 
5]. However, the quest for sufficiently accurate equilibrium $\mathrm{C}-\mathrm{C}$ bond lengths poses a challenge to both experiment and theory.

On the experimental side, one faces difficulties in obtaining good-quality monocrystals. Moreover, the usual diffraction techniques (by X-rays or neutrons) suffer from the limitations of the basic theoretical model which translates the diffraction data into the molecular geometry. This model, in which spherical atoms are subject to uncorrelated thermal motions, neglects (i) the changes of the electronic density due to chemical bonding (contributing to the $\mathrm{X}$-ray diffraction), as well as (ii) the rigid-body motions of the whole molecule. The latter effects, affecting both the X-ray and neutron studies, are especially important in the case of flat and rigid structures of PAHs. The influence of factors (i) and (ii) on the equilibrium $\mathrm{C}-\mathrm{C}$ bond lengths is well documented in the X-ray studies of naphthalene and anthracene by Brock et al. [7]. Nevertheless, ample raw X-ray and neutron structural data provide a basis for meaningful chemometric studies of bond lengths in PAHs; see papers of Kiralj and Ferreira [6], Kiralj et al. [8], and references therein. Such studies allow a glimpse into another factor affecting the molecular $\mathrm{C}-\mathrm{C}$ bond lengths derived from crystal structural data: (iii) the influence of crystal-packing forces [6].

On the theory side, there are convergence problems of quantum-mechanical calculations with respect to (i) orbital basis sets and (ii) electronic-correlation contributions. Moreover, full geometry optimization becomes quite costly for big molecules. One finds these problems especially acute in calculations for $\pi$-electron systems, PAHs in particular. The density functional theory (DFT) seems to be the ab initio method of choice for the geometry optimization of such molecules as PAHs. In the study of acenes (from naphthalene $\mathrm{C}_{10} \mathrm{H}_{8}$ to hexacene $\mathrm{C}_{26} \mathrm{H}_{16}$ ) by Kadantsev et al. [9], the geometries of these molecules were optimized by using three popular variants of approximate DFT functionals (SVWN5, PBE, and B3LYP), and a big Gaussian basis set $(6-311++G(d, p))$. The comparison of the equilibrium C-C bond lengths calculated by means of the SVWN5, PBE, and B3LYP functionals reveals differences which often exceed $0.01 \AA$. One has to concede that "benchmark" geometries of even relatively small PAHs (up to 26 carbon atoms) have not been yet established.

As it has been already in the past, a carefully crafted semi-empirical quantum approach may come to a rescue. In Refs. [4, 5], a very simple Augmented Hückel Molecular Orbital (AugHMO) model has been designed for this purpose. It allows for a qualitative, as well as quantitative, study of the bond-length variation in general $\pi$-electron molecules (i.e., those which are planar or locally planar). The simplest realization of the AugHMO model, the Hückel-Su-Schrieffer-Heeger (HSSH) method, was parametrized [5] for the $\pi$-electron hydrocarbons and all-carbon molecules. A comparison with available experimental data demonstrated [5] that the HSSH method is capable of a good description of the C-C bond lengths in a variety of $\pi$-electron hydrocarbons and carbon systems, including fullerene $\mathrm{C}_{60}$, polyacetylene, and graphene. It was also found [5] that the HSSH results use to be quite similar to those calculated by means of the B3LYP functional with a modest Gaussian basis set (6-31G(d)). Interestingly, for acenes, the C-C bond lengths calculated by means of the B3LYP/6-31G(d) approach by Firouzi and Zahedi [10] are in almost perfect agreement with those calculated by Kadantsev et al. [9] by means of the B3LYP/6-311++G(d,p) approach. It should be also stressed that, in contrast to ab initio methods, the HSSH method of Ref. [5] can be applied to PAHs containing thousands of carbon atoms.

In the present paper, we apply the HSSH method to calculate the equilibrium C-C bond lengths for the set of 34 polycyclic aromatic PAHs presented in the Riegel and Müllen paper [3]. In our study, we keep the classification of PAHs, as well as the numbering of the molecules, employed in Ref. [3]:

- Clarenes (i.e., fully benzenoid hydrocarbons [11, 12]): molecules 1-14, see "Clarenes," Figs. 2 and 3.

- K-region PAHs: molecules 15-20 (plus ovalene), see "K-Region PAHs," Figs. 4 and 5.

- Phenacenes: molecules 21-25, see "Phenacenes," Fig. 6.

- Rylenes: molecules 26-29, see "Rylenes," Fig. 7.

- Acenes: molecules 30-34 (plus naphthalene), see “Acenes," Fig. 8.

\section{HSSH calculations}

The HSSH bond-length optimization procedure is a (simplified) analog of the molecular-geometry optimization techniques that employ the analytical gradients and Hessians of the total molecular energy [13]. In the HSSH method, the hydrocarbon under study is fully characterized by its topology of C-C bonds (which is coded in the form of the topological matrix $[4,5])$.

At the start of the calculations, all the $\mathrm{C}-\mathrm{C}$ bonds are put equal to the value for benzene, $R_{\text {ben }}^{\mathrm{e}}=1.397 \AA$. Then, the usual Hückel calculations follow, yielding the $\pi$ molecular orbitals ( $\pi$ MOs) and the $\pi$-electron bond orders $p_{\text {bond }}$. This is the 0th iteraction, fully equivalent to the standard Hückel approach. In each subsequent step (iteration), the condition of the vanishing gradient of the total HSSH energy is enforced: this amounts to iterating the linear bond-order bond-length (BO-BL) relationship:

$R_{\text {bond }}^{\mathrm{e}}=R^{\mathrm{o}}-x p_{\text {bond }}^{\mathrm{e}}$, 
where $R_{\text {bond }}^{\mathrm{e}}$ and $p_{\text {bond }}^{\mathrm{e}}$, respectively, are the equilibrium bond length and the $\pi$-electron bond order for a given C-C bond. $R^{\mathrm{o}}=1.523 \AA$ and $x=0.189 \AA$ are two geometrical parameters of the AugHMO model of $\pi$ electron hydrocarbons $[4,5]$. In the HSSH method for hydrocarbons [5], there is also a third geometrical parameter $y=0.2756 \AA$ which determines the slope of a linear function $\beta(R)$ describing the dependence of the "resonance integral" $\beta(<0)$ on the C-C bond length $R$. In order to improve the rate of convergence of the iterative procedure, the Hessian of the HSSH total energy is calculated and applied [4]. In our calculations for PAHs, we assumed that the iterations stopped when the subsequent values of bondlength differences dropped below $5 \cdot 10^{-6} \AA$. To this end, five "direct iterations" using Eq. 1 followed by two to three iterations involving the Hessian were always sufficient.

Some clarifications concerning the application of the HSSH method $[4,5]$ to PAHs are in order:

(I) This method optimizes only the bond lengths, and it is assumed that the optimal valence angles (the C$\mathrm{C}-\mathrm{C}$ ones in the case of PAHs) are consistent with the optimized C-C bond lengths, and are as close to $120^{\circ}$ as possible. Reducing the molecular geometry solely to the C-C bond lengths seems justified for such molecules as PAHs, and contribute to the computational effectiveness of the HSSH method.

(II) While Eq. 1 looks like a relationship of local character, it should be remembered that the $\pi$-electron bond orders $p_{\text {bond }}$ are derived from the set of completely delocalized occupied $\pi$ molecular orbitals [4]. And in the HMO model (as well as in the AugHMO one), these bond orders appear to be sensitive probes of molecular topology. When this is of interest, one may use Eq. 1 to recover the values of the equilibrium $\pi$-electron bond orders $p_{\text {bond }}^{\mathrm{e}}$ from the corresponding calculated equilibrium $\mathrm{C}-\mathrm{C}$ bond lengths $R_{\text {bond }}^{\mathrm{e}}$. BO-BL relationships of the linear (and also of the quadratic) form were extensively used by Kiralj et al. [8] in order to relate the experimental C-C bond lengths, obtained from crystal diffraction studies, to the theoretical bond orders calculated by using various theoretical methods. That methodology is different from the one employed in the present paper, but in some cases (a linear BO-BL relationship of the form of Eq. 1, bond orders corresponding to the SCF and VSCF methods [8]), their linear regression coefficients were quite close to our values of $R^{\mathrm{o}}$ and $x$.

(III) PAHs belong to the class of alternant $\pi$-electron hydrocarbons [5], and within the HMO (AugHMO) model the Coulson-Rushbrooke theorem [14] holds: (i) The $\pi$-electron orbital energies for the occupied and unoccupied states are placed symmetrically with respect to the value of the "Coulomb integral" $\alpha$, (ii) the net $\pi$-electron charges [4] at the $\mathrm{C}$ atoms are equal to 0 .

(IV) Every PAH contains an even number of $\pi$ electrons $\left(N^{\pi}=2 n\right)$, equal to the number of carbon atoms $(M=2 n)$. The ground state of the PAH molecule involves $n$ doubly occupied $\pi$ MOs of lowest orbital energies, and thus corresponds to the closed shell (the spin singlet). In our calculations, we monitor the HOMO-LUMO gap (nonzero), and the smallest eigenvalue (positive) of the Hessian of the total HSSH energy.

(V) In the HSSH calculations for hydrocarbons, we employ the Hückel energy units:

$\alpha=0, \quad|\beta| \equiv\left|\beta\left(R_{\text {ben }}^{\mathrm{e}}\right)\right|=1$,

where $R_{\text {ben }}^{\mathrm{e}}=1.397 \AA$ serves as the reference C-C bond length [5].Thus, the only empirical parameters of the HSSH model are the abovementioned three geometrical parameters: $R^{\mathrm{o}}, x$, and $y$, plus $R_{\text {ben }}^{\mathrm{e}}$.

\section{HOMA index}

We used our HSSH-optimized C-C bond lengths to calculate the values of the aromaticity index HOMA of Krygowski and coworkers [15-18]. HOMA (HarmonicOscillator Measure of Aromaticity) is currently considered the most important indicator of the aromatic character of $\pi$ electron molecules (or their fragments), based solely on the values of bond lengths. For a $\pi$-electron hydrocarbon, the definition of HOMA reads as $[15,16]$

$\mathrm{HOMA}=1-\frac{\kappa}{N} \sum_{\text {bond }}\left(R_{\text {bond }}^{\mathrm{e}}-R_{\text {opt }}\right)^{2}$,

where the summation runs over all $N$ carbon-carbon bonds in the $\pi$-electron hydrocarbon (or its fragment), $\kappa=257.7$ $\AA^{-2}$ is a normalization constant, while $R_{\text {opt }}=1.388 \AA$ represents the optimal value of the $\mathrm{C}-\mathrm{C}$ bond length in the HOMA model. Although of energetic provenience [15], HOMA of Eq. 3 is dimensionless and fulfils condition $0 \leq$ HOMA $\leq 1$ (smaller HOMA indicates lower aromatic character). It was found $[17,18]$ that definition (3) can be rewritten in a more revealing form:

$$
\begin{aligned}
\mathrm{HOMA}= & 1-\mathrm{EN}-\mathrm{GEO}=1-\kappa\left(R_{\mathrm{av}}-R_{\mathrm{opt}}\right)^{2} \\
& -\frac{\kappa}{N} \sum_{\text {bond }}\left(R_{\mathrm{bond}}^{\mathrm{e}}-R_{\mathrm{av}}\right)^{2},
\end{aligned}
$$

where

$R_{\mathrm{av}}=\frac{1}{N} \sum_{\text {bond }} R_{\mathrm{bond}}^{\mathrm{e}}$

is the average $\mathrm{C}-\mathrm{C}$ bond length in the $\pi$-electron hydrocarbon (or its fragment). The components EN and GEO 
may be interpreted as some "dearomatization" contributions originating from the bond-length elongation and bond alternation, respectively [18]. Let us note that the EN component is simply a quadratic function of $R_{\mathrm{av}}$, and that the EN and GEO components (and thus HOMA) are sensitive to the rounding errors in the values of $R_{\mathrm{bond}}^{\mathrm{e}}$.

\section{Modular architecture of PAHs}

The carbon skeletons of PAHs are finite jigsaw cuts from the honeycomb lattice of graphene. At the perimeter of a PAH, there is a number of methine groups $(>\mathrm{CH})$, corresponding to the $\mathrm{C}$ atoms with two $\mathrm{C}$ neighbors; of all the remaining $\mathrm{C}$ atoms, each has three $\mathrm{C}$ neighbors. The varied shapes of PAHs determine the corresponding topologies of the C-C bonds, which may be represented by the so-called Hückel graphs (see Ref. [19], p. 28). We shall use such graphs in the figures throughout the paper: the $\mathrm{C}$ atoms are graph vertices, and the $\mathrm{C}-\mathrm{C} \sigma$ bonds are graph edges (the $\mathrm{H}$ atoms and the $\mathrm{C}-\mathrm{H}$ bonds are absent). In the AugHMO model [4, 5], the topologies of the $\mathrm{C}-\mathrm{C}$ bonds translate into the bondlength patterns. A practical realization of that model, the HSSH method described in "HSSH calculations," is used here exactly in the same manner as ab initio methods with full geometry optimization [13]: with no constraints, scaling factors, external corrections, etc. Therefore, the final results (the equilibrium C-C bond lengths) and their analysis are not subject to some preconceived notions.

Our HSSH calculations for PAHs reveal a considerable spread (of ca. $0.1 \AA$ ) of their equilibrium C-C bond lengths: from $1.363 \AA$ (bond a in pyrene (15), see Fig. 4) to 1.461 $\AA$ (bond m' in perylene (26), see Fig. 7). These values are to be compared with the HSSH result for the C-C bond in graphene [5], equal to $1.424 \AA$.

A careful and unbiased analysis of all the calculated C-C bond lengths has led us to the following observation: some classes of PAHs (clarenes, rylenes, and a subclass of the Kregion PAHs) are well represented by modular architecture. In particular, the equilibrium ground-state geometry of a PAH belonging to one of these classes can be assembled from certain standard molecular fragments (modules) of fixed geometries, connected by C-C bonds (linkers) of fixed lengths. The set of modules employed in the present study is depicted, in the form of Hückel graphs, in Fig. 1. These modules are assumed to have "natural" symmetries, independent of the particular molecular environment. More details will be provided in sections devoted to clarenes ("Clarenes"), K-region PAHs (2) ("K-Region PAHs (2): "superbenzene" derivatives"), and rylenes ("Rylenes").

How are the bond lengths corresponding to the modules and linkers computed? Take the example of bond a in module A3: the length $\overline{R_{\mathrm{a}}^{\mathrm{e}}}$ was calculated by averaging over all the 28 symmetry-unrelated occurrences of this type of bond in the molecules containing the A3-module (see Figs. 2, 3, and 5). As a measure of the spread of the actual values of this bond length, we used the absolute value of a maximal difference:

$\Delta_{a}=\max \left|R_{\mathrm{a}}^{\mathrm{e}}-\overline{R_{\mathrm{a}}^{\mathrm{e}}}\right|$,

corresponding to the abovementioned set of 28 values of $R_{\mathrm{a}}^{\mathrm{e}}$.

\section{Clarenes}

Eric Clar proposed $[11,12]$ that the fully benzenoid hydrocarbons are special because of being ideal superpositions of six-electron units-the "Clar sextets." Thus, it seems quite proper to call this class of PAHs "clarenes." The family of clarenes taken from Ref. [3] consists of 14 molecules, for convenience split into two subsets shown in Figs. 2 and 3.

Every molecule of clarene contains $6 \mathrm{~N}$ carbon atoms and can be partitioned into $N$ six-carbon-atom benzene-like fragments, hereafter called the A-modules. Each A-module carries six $\pi$ electrons-this is the abovementioned Clar sextet. Within a given molecule, the A-modules (depicted in Fig. 1) are connected by $\mathrm{C}-\mathrm{C}$ bonds, hereafter called the AA-linkers. The An-module ( $\mathrm{n}=2,3,4,5,6)$ uses $\mathrm{n}$ linkers to connect with other modules; benzene may be considered a "honorary" A0-module, while the phenyl group (usually not appearing in PAHs) is the A1-module. Modules A4 and A4' correspond to two different arrangements of four linkers. Moreover, linkers connecting the same pair of modules (e.g., A3-A5) may correspond to different linking topologies: see, e.g., linker u in molecule 12, and linkers u' and $\mathrm{u}^{\prime}$ ' in molecule 6.

Within a clarene molecule, the A-modules correspond to the so-called "full" (hexagonal) carbon rings, while those hexagonal rings which contain (three) linkers are called "empty" rings [11]. Our HSSH calculations demonstrate that, with sufficient accuracy, each clarene can be additively assembled from standard A-modules, connected via some standard AA-linkers. The results in Tables 1 and 2 are quite convincing: the An-modules ( $\mathrm{n}=2-5$ ) have welldefined geometries (with characteristic bond lengths), and the AA-linkers of a given type have nearly constant lengths. It is seen that within the A-modules the bond lengths are distinctly shorter than those corresponding to the AAlinkers. Clearly, this is an indication that the Clar sextets are, to some degree, localized within the A-modules (i.e., the "full" rings).

The An-modules ( $\mathrm{n}=2-5$ ) necessarily have to be located at the perimeter of a given clarene. The interior 
Fig. 1 A-, B-, and C-modules. Hückel graphs, with symbols of symmetry nonequivalent bonds; directions of linkers (with no endpoint $\mathrm{C}$-atoms) are indicated<smiles>CC1CCCCC1C</smiles>

A2
A3<smiles>CC1CCCC(C)C1C</smiles><smiles>CC1CC(C)C(C)C(C)C1C</smiles>

A5<smiles>CC1[C]CCC2CCCC(C)C12</smiles>

B2
A4<smiles>CC1[C]CC(C)C(C)C1C</smiles>
A4'
A6<smiles>CC1C(C)C(C)C(C)C(C)C1C</smiles>

a<smiles>CC1CCC(C)C2C(C)CCC(C)C12</smiles>

B4<smiles>CC1CC(C)C(C)CC1C</smiles><smiles>CC1CCC2CCC3CCC(C)C(C)C3C2C1C</smiles>

of the molecule, if sufficiently big, is filled with the A6modules. Quite surprisingly, A6 cannot be considered a rigid building block: in a less symmetric surrounding, its perfect $6 \mathrm{~mm}\left(\mathcal{C}_{6 v}\right)$ symmetry ${ }^{1}$ becomes visibly perturbed. The most important cases of $\mathbf{A 6}$ deformations are presented in Table 3. In molecules $\mathbf{1 1}$ and $\mathbf{2 0}$ (see Fig. 5), the molecular symmetry group $3 m\left(\mathcal{C}_{3 v}\right)$ induces some bond alternation in the central A6-module; however, this effect is absent in molecule $\mathbf{1 4}$ due to a different orientation of the molecular-symmetry elements with respect to the central A6 hexagon. A similar pattern of deformation is found in molecule 19, despite its lower symmetry corresponding to the $m\left(\mathcal{C}_{\mathrm{s}}\right)$ group. It is apparent that bond alternation is the softest mode of deformation of the A6-module.

It should be noted that in a PAH molecule all the carbon atoms having three $\mathrm{C}$ neighbors are completely inert [20], and thus the A6-modules do not contribute to chemical

\footnotetext{
${ }^{1}$ We use here the symbols corresponding to the planar point groups [5]
}

reactivity-all the chemistry takes place at the perimeter of the molecule, involving the methine groups of the Anmodules with $n=2-5$.

The inspection of the HOMA values and its components in Table 4 indicates that the An-modules retain, to some degree, the aromaticity of the benzene molecule. It is seen that it is $\mathrm{EN}$ (and thus $\mathrm{R}_{\mathrm{av}}$ ) which is mostly responsible for diminishing of HOMA with the increase of $n$ (the number of linkers).

\section{On the path from clarenes to graphene}

In a series of papers, Tyutyulkov, Müllen, and their collaborators [21-24] posed a question: "is graphene an ultimate large hydrocarbon?" In order to find the answer, they analyzed various aspects: structural (topology), energetic (the energy spectra), and the influence of defects (including these with unpaired spins) and different edge structures. Their findings suggest [21] that the electronic-correlation effects should lead to a nonzero gap in the energy spectrum 


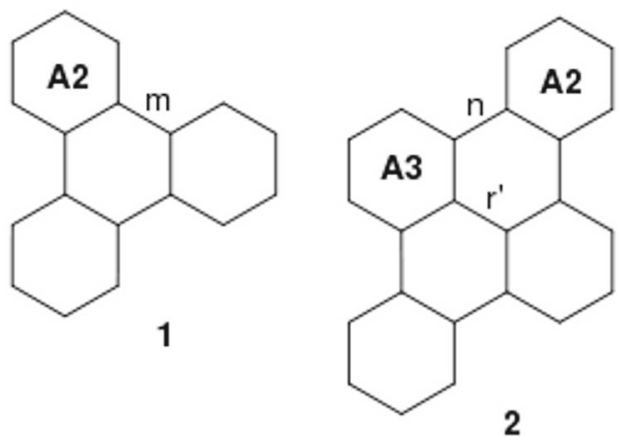

2

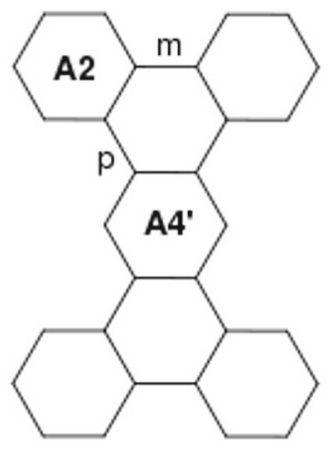

3

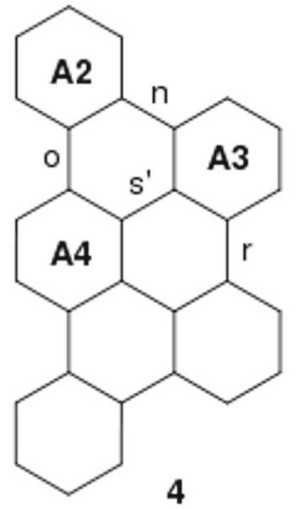

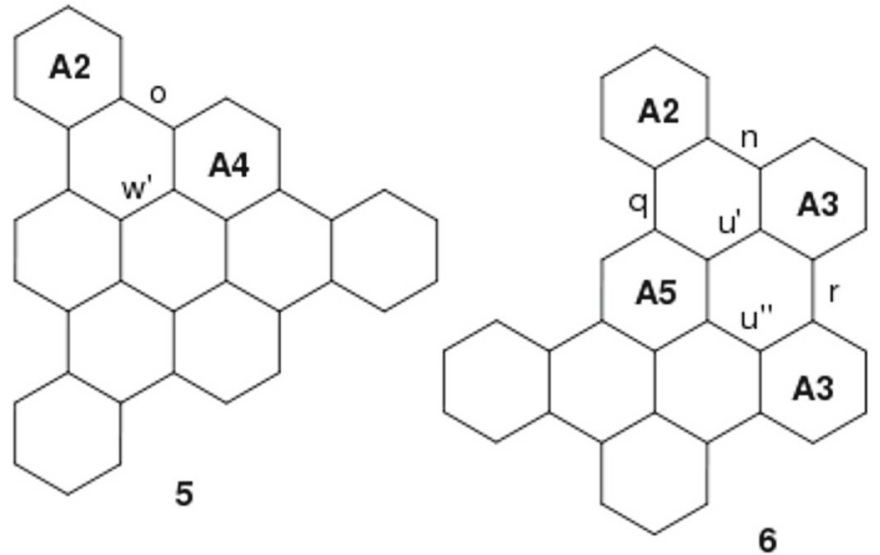

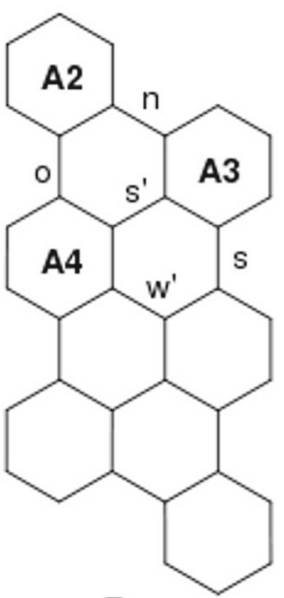

7

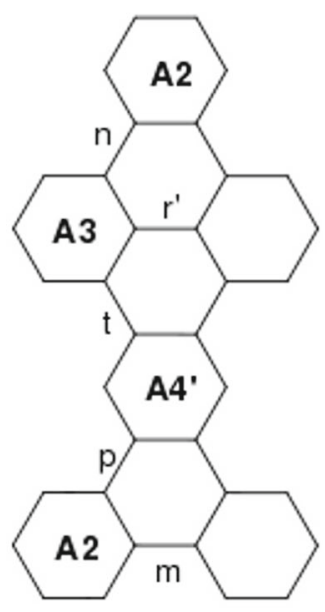

8

Fig. 2 Clarenes (1). Hückel graphs, with symbols of symmetry nonequivalent A-modules and AA-linkers

of PAHs, even in the limit of an infinite molecule (thus, strictly speaking, the metallic limit is not reached).

We are going to use our HSSH results for clarenes to approach the above question from the perspective of molecular geometries: the $\mathrm{C}-\mathrm{C}$ bond lengths, and also the HOMA values derived from them.

The molecular values of HOMA and its components collected in Table 5 show that the aromatic character of clarenes is lowering with their size. Both the EN and GEO components contribute to this effect. As clarenes grow larger, one finds that (i) the value of $R_{a v}$ increases and thus departs from the HOMA value of $\mathrm{R}_{\mathrm{opt}}$, and (ii) the HOMO-LUMO gap (as calculated within the HSSH model) diminishes. Even if big clarenes are still far from the graphene limit, one may speculate that the metallic character and aromaticity are in conflict. The HOMA result for graphene suggests its low aromaticity, despite the fact that graphene is the most stable of all $\pi$-electron hydrocarbon and carbon molecules (as the molecular enthalpies of formation attest).
The modular architecture of clarenes, uncovered in the course of our HSSH calculations, provides further insights. It is seen that the perimeter of a clarene assumes a definite geometric structure corresponding to some arrangement od the An-modules $(\mathrm{n}=2-5)$. On the other hand, for very big clarenes, their interior is expected to approach the geometric structure of (infinite) graphene. To this end, the equilibrium bond lengths corresponding to bonds a and a' in the A6-module (see Fig. 1), and the equilibrium bond length corresponding to the A6-A6 linker (z", see molecule 14 in Fig. 3) should approach the HSSH value for graphene (1.424 $\AA$ ).

For the largest PAH in this study, molecule 14 (96 C atoms), we found $R_{\mathrm{a}}^{\mathrm{e}}=R_{\mathrm{a}}^{\mathrm{e}}=1.419 \AA, R_{\mathrm{z}}^{\mathrm{e}},=1.435$ $\AA$, quite far from the graphene limit. However, when one averages over three bond lengths adjacent to any $\mathrm{C}$ atom in any A6-module, the result is very close to $1.424 \AA$. Our tentative conclusion from the above observation is as follows: even in relatively small clarenes, their interiors "prepare" for becoming graphene-like. 


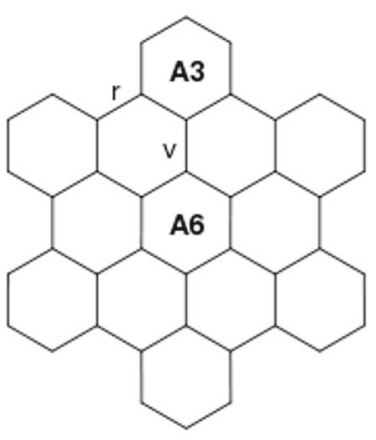

9

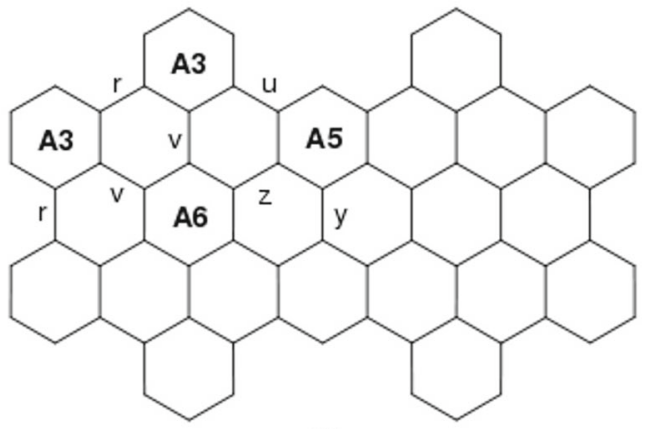

12

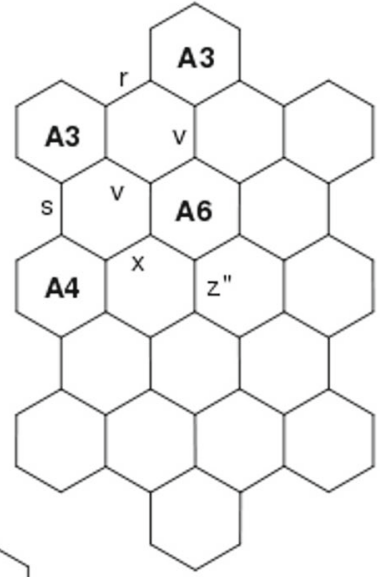

10

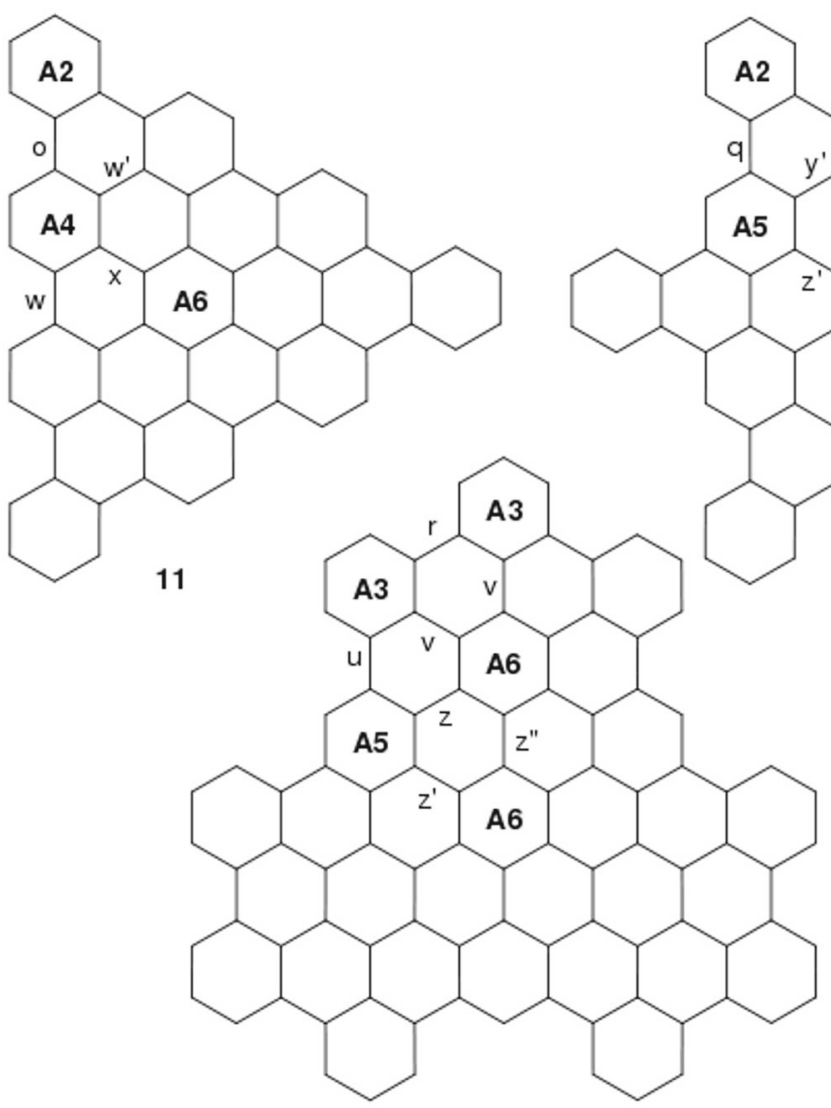

14

Fig. 3 Clarenes (2). Hückel graphs, with symbols of symmetry nonequivalent A-modules and AA-linkers

Table 1 A-modules (up to A5). HSSH equilibrium C-C bond lengths (in $\AA$ )

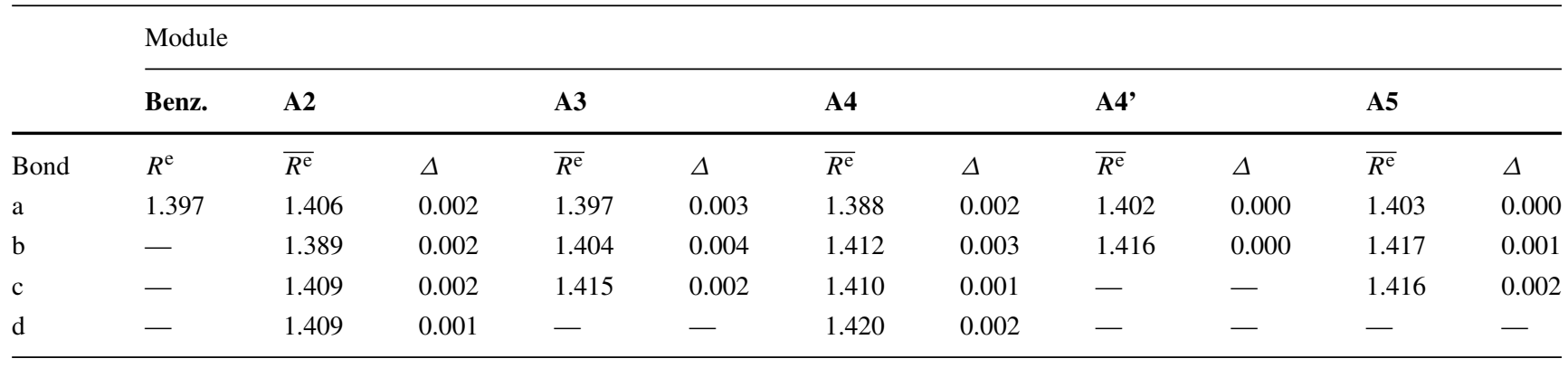


Table 2 AA-linkers. HSSH equilibrium C-C bond lengths (in $\AA$ )

\begin{tabular}{llllll}
\hline Linker & $\overline{R^{\mathrm{e}}}$ & $\Delta$ & Linker & $\overline{R^{\mathrm{e}}}$ & \multicolumn{1}{c}{$\Delta$} \\
\hline m (A2-A2) & 1.456 & 0.001 & w (A4-A4) & 1.447 & - \\
n (A2-A3) & 1.455 & 0.001 & w' (A4-A4) & 1.442 & 0.001 \\
o (A2-A4) & 1.452 & 0.002 & x (A4-A6) & 1.439 & - \\
p (A2-A4') & 1.456 & 0.000 & & & \\
q (A2-A5) & 1.454 & 0.001 & & & \\
r (A3-A3) & 1.455 & 0.001 & y (A5-A5) & 1.438 & - \\
r' (A3-A3) & 1.444 & 0.000 & y' (A5-A5) & 1.447 & - \\
s (A3-A4) & 1.452 & 0.001 & z (A5-A6) & 1.437 & 0.001 \\
s' (A3-A4) & 1.443 & 0.001 & z' (A5-A6) & 1.436 & 0.000 \\
t (A3-A4') & 1.456 & - & & & \\
u (A3-A5) & 1.453 & 0.000 & & & \\
u' (A3-A5) & 1.442 & - & & & \\
u”(A3-A5) & 1.443 & - & & & \\
v (A3-A6) & 1.440 & 0.003 & z" (A6-A6) & 1.436 & 0.002 \\
\hline
\end{tabular}

We thus propose, with some exaggeration, that a big clarene molecule has a "hard shell" made of some Anmodules $(\mathrm{n}=2-5)$, protecting an interior consisting of "softer" A6-modules, which, with the growing size of the molecule, loose their identities and, finally, melt into a homogenous honeycomb lattice of graphene. In the language of the Clar sextet concept [11], one may say that, on the path to the graphene limit, the "full" and "empty" rings in the clarene interior become indistinguishable. There is a consonant observation coming from the theoretical studies of (topological) ring currents in clarenes by Dickens and Mallion [25, 26]. In Ref. [26], the authors stated that "... as the number of rings in the structure increases, the ... sharp distinction between 'full' and 'empty' rings begins to break down, as far as ring currents are concerned."

While experimental and theoretical studies of graphene focus on its interior, it should be remembered that every real graphene specimen has a boundary, involving dangling bonds or some substituted atoms (hydrogens, for example). In particular, such a boundary may have a form of some regular perimeter consisting of the methine groups, as in the case of clarenes or K-region PAHs of "K-Region PAHs."

Table 3 A6-modules, HSSH equilibrium C-C bond lengths (in $\AA$ )

\begin{tabular}{lllll}
\hline Molecule & $R_{\mathrm{a}^{\prime}}^{\mathrm{e}}(\AA)$ & $R_{\mathrm{a}}^{\mathrm{e}}(\AA)$ & $\frac{1}{2}\left(R_{\mathrm{a}^{\prime}}^{\mathrm{e}}+R_{\mathrm{a}}^{\mathrm{e}}\right)$ & $R_{\mathrm{a}}^{\mathrm{e}}-R_{\mathrm{a}}^{\mathrm{e}}$ \\
\hline $\mathbf{1 1}$ & 1.420 & 1.414 & 1.417 & 0.006 \\
$\mathbf{1 9}$ & 1.421 & 1.414 & 1.417 & 0.007 \\
$\mathbf{2 0}$ & 1.424 & 1.413 & 1.419 & 0.010 \\
\hline
\end{tabular}

In molecules 11, 19, and 20, a pattern of alternating bonds (a' a) 3 is seen. Full averaging over the remaining molecules containing A6-modules gives $R_{\mathrm{a}^{\prime}}^{\mathrm{e}}=R_{\mathrm{a}}^{\mathrm{e}}=1.417, \Delta=0.003$
Table 4 A-Modules. $\mathrm{R}_{\mathrm{av}}$, EN, GEO, and HOMA values

\begin{tabular}{lllll}
\hline Module & $\mathrm{R}_{\mathrm{av}}(\AA)$ & EN & GEO & HOMA \\
\hline Benzene & 1.397 & 0.021 & 0.000 & 0.979 \\
$\mathbf{A 2}$ & 1.402 & 0.051 & 0.021 & 0.928 \\
$\mathbf{A 3}$ & 1.405 & 0.076 & 0.013 & 0.911 \\
$\mathbf{A 4}$ & 1.409 & 0.110 & 0.043 & 0.847 \\
$\mathbf{A 4}$, & 1.406 & 0.086 & 0.012 & 0.902 \\
$\mathbf{A 5}$ & 1.412 & 0.150 & 0.011 & 0.839 \\
A6 & 1.417 & 0.217 & 0.000 & 0.783 \\
\hline
\end{tabular}

Therefore, studies of giant PAHs are of utmost relevance to the science of graphene.

\section{K-Region PAHs}

Riegel and Müllen [3] coined the name "K-region PAH" to denote the PAH which can be derived from a clarene having one or more "bays" within its perimeter. The "bay" consists of four $\mathrm{C}$ atoms arranged as in the s-cis butadiene molecule (at the "mouth" of the bay there are two methine groups, causing some steric tension). Examples of such bays are seen in clarenes 1 - 14. The K-region emerges when the mouth of the bay is closed with a two-carbon insertion of the formula $-\mathrm{CH}-\mathrm{CH}-$; we shall call this insertion the "fhandle," alluding to the bond $\mathrm{f}$ in phenanthrene (21); see Fig. 6. Obviously, the new molecule does not fit the clarene paradigm, and the K-region (corresponding to the f-handle) introduces some olefinic character to the molecule (for a

Table 5 Clarenes. Molecular values of $\mathrm{R}_{\mathrm{av}}$ (in $\AA$ ), EN, GEO, and HOMA

\begin{tabular}{lllll}
\hline Molecule & $\mathrm{R}_{\mathrm{av}}(\AA)$ & EN & GEO & HOMA \\
\hline $\mathbf{1}$ & 1.409 & 0.119 & 0.106 & 0.775 \\
$\mathbf{2}$ & 1.412 & 0.147 & 0.105 & 0.748 \\
$\mathbf{3}$ & 1.412 & 0.143 & 0.115 & 0.742 \\
$\mathbf{4}$ & 1.413 & 0.164 & 0.103 & 0.733 \\
$\mathbf{5}$ & 1.414 & 0.175 & 0.099 & 0.727 \\
$\mathbf{6}$ & 1.414 & 0.176 & 0.102 & 0.722 \\
$\mathbf{7}$ & 1.414 & 0.175 & 0.100 & 0.725 \\
$\mathbf{8}$ & 1.413 & 0.158 & 0.112 & 0.730 \\
$\mathbf{9}$ & 1.416 & 0.199 & 0.090 & 0.711 \\
$\mathbf{1 0}$ & 1.417 & 0.219 & 0.080 & 0.701 \\
$\mathbf{1 1}$ & 1.416 & 0.208 & 0.086 & 0.706 \\
$\mathbf{1 2}$ & 1.417 & 0.222 & 0.082 & 0.696 \\
$\mathbf{1 3}$ & 1.417 & 0.214 & 0.086 & 0.700 \\
$\mathbf{1 4}$ & 1.418 & 0.238 & 0.073 & 0.689 \\
Graphene & 1.424 & 0.334 & 0.000 & 0.666 \\
\hline
\end{tabular}


Fig. 4 K-region PAHs (1): pyrene, coronene, and ovalene. Hückel graphs, with symbols of symmetry nonequivalent bonds a<smiles>CC12CCCC3CCC4CCC(C1)C4C32</smiles>

Pyrene (15) detailed discussion on the chemical and spectral properties of the K-region PAHs, see Ref. [3]).

The K-region PAHs of Ref. [3] may be conveniently divided into two groups: one containing pyrene and coronene (and ovalene, added here), and second composed of molecules which may be derived from "superbenzene" (9), see Fig. 3.

\section{K-Region PAHs (1): pyrene, coronene, and ovalene}

Pyrene, coronene, and ovalene, depicted in Fig. 4, are wellknown PAHs. Their C-C bond lengths are listed in Table 6: a closer inspection of these data indicates that no obvious transferable fragments (modules) can be identified. This is a striking contrast with the clarenes of the previous section. One can identify the "olefinic" f-handles as the a-bonds in all three molecules, and the f-bond in ovalene.

The molecular values of HOMA and its components are collected in Table 7. The general trends are similar to those for clarenes (see Table 5), with the GEO contributions being slightly larger. Let us note that HOMA for coronene is much lower than that for benzene, and not very different from that of graphene (see Table 4).

Table 6 Pyrene, coronene, and ovalene. HSSH equilibrium C-C bond lengths (in $\AA$ )

\begin{tabular}{llll}
\hline Bond & Pyrene & $\begin{array}{l}\text { Molecule } \\
\text { Coronene }\end{array}$ & Ovalene \\
\hline $\mathrm{a}$ & 1.363 & 1.374 & 1.382 \\
$\mathrm{~b}$ & 1.444 & 1.429 & 1.419 \\
$\mathrm{c}$ & 1.418 & 1.411 & 1.412 \\
$\mathrm{~d}$ & 1.432 & 1.430 & 1.425 \\
$\mathrm{e}$ & 1.406 & - & 1.438 \\
$\mathrm{f}$ & 1.397 & - & 1.367 \\
$\mathrm{~g}$ & - & - & 1.440 \\
$\mathrm{~h}$ & - & - & 1.405 \\
$\mathrm{i}$ & - & - & 1.422 \\
$\mathrm{j}$ & - & - & 1.432 \\
$\mathrm{k}$ & - & - & 1.417 \\
$\mathrm{l}$ & - & - & 1.438 \\
\hline
\end{tabular}

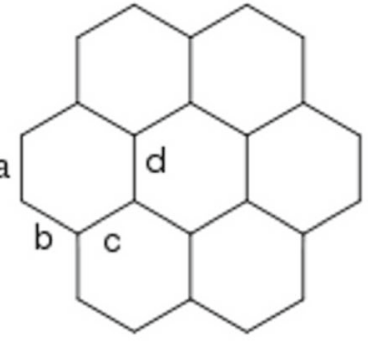

Coronene (16)

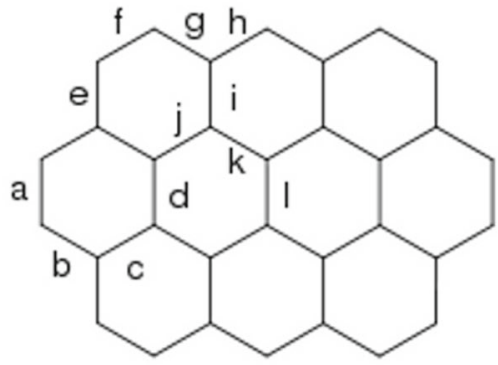

Ovalene

\section{K-Region PAHs (2): "superbenzene" derivatives}

The second group of the K-region PAHs of Ref. [3] can be derived by inserting the f-handles into the bay regions of clarene 9, called [27] "superbenzene" (according to that nomenclature, clarene $\mathbf{1 2}$ is "supernaphthalene," and clarene $\mathbf{1 4}$ is "superphenalene"). One insertion gives molecule 17, two (different) insertions-molecules 18 and 19, and three insertions-molecule 20 (see Fig. 5).

In molecules $\mathbf{1 7}$ - $\mathbf{2 0}$ one may identify the A3- and A6-modules corresponding to clarene 9, with practically "frozen" structures (see Table 2) (also linkers $r$ and $\mathrm{v}$ are unchanged). But a new module can be identified: this is the C4-module (see Fig. 1), involving fourteen $\mathrm{C}$ atoms (and fourteen $\pi$ electrons), which resembles the phenanthrene molecule (21), a "honorary" C0-module. As seen in Table 8, the (averaged) equilibrium C-C bond lengths of the C4-module are pretty well transferable, and so are the corresponding linkers (linker $\mathrm{k}$ being here a bit of exception).

The values of HOMA and its components for phenanthrene and the C4-module are collected in Table 9. The molecular values of HOMA and its components presented in Table 10 show that each insertion of the f-handle into clarene lowers its HOMA, and thus diminishes its aromatic character.

\section{Phenacenes}

The first five members of the family of phenacenes are shown in Fig. 6. With the growing size, they approach

Table 7 Pyrene, coronene, and ovalene. Molecular values of $\mathrm{R}_{\mathrm{av}}$, EN, GEO, and HOMA

\begin{tabular}{lllll}
\hline Molecule & $\mathrm{R}_{\mathrm{av}}(\AA)$ & EN & GEO & HOMA \\
\hline Pyrene & 1.411 & 0.141 & 0.142 & 0.717 \\
Coronene & 1.414 & 0.179 & 0.118 & 0.703 \\
Ovalene & 1.416 & 0.203 & 0.118 & 0.679 \\
\hline
\end{tabular}


Fig. 5 K-Region PAHs (2).

Hückel graphs, with symbols of symmetry nonequivalent $\mathrm{A}$ - and C-modules, and linkers

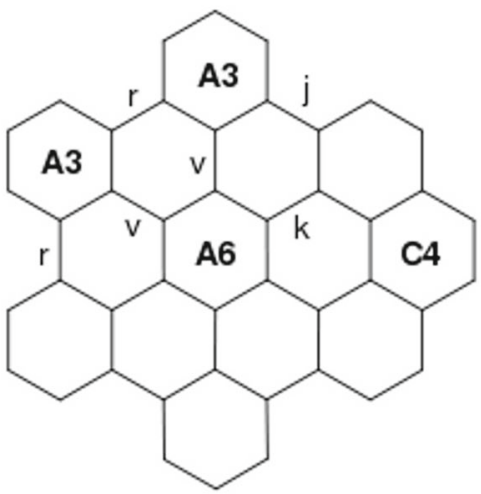

17

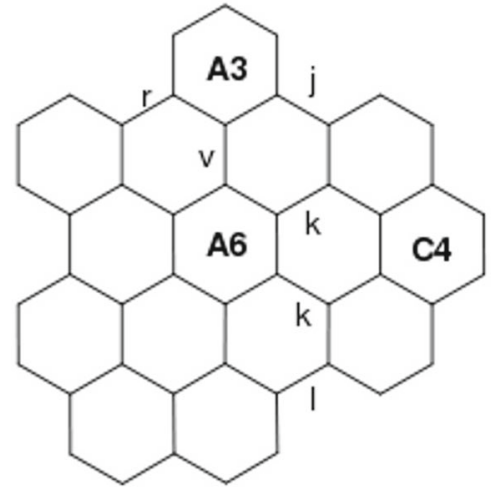

19

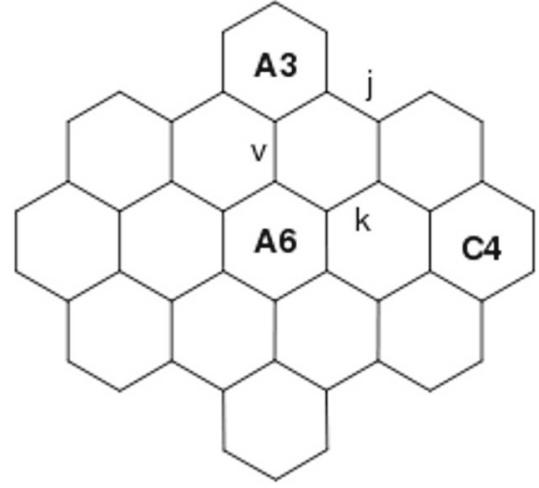

18

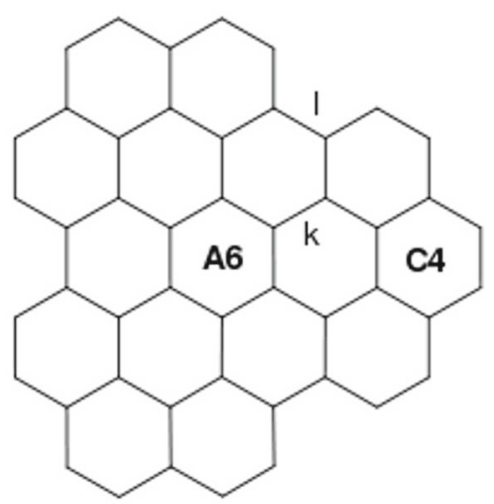

a limit of a $\pi$-electron polymer (not the $2 \mathrm{D}$ structure of graphene). The bond lengths of phenacenes are presented in Table 11: here also we see no clue to identify transferable modules, although some regularities in bond lengths are clearly visible.

Our HSSH equilibrium C-C bond lengths in phenacenes may be compared with those calculated by Firouzi and

Table 8 C-Modules and AC-and CC-linkers. HSSH equilibrium C-C bond lengths (in $\AA$ )

\begin{tabular}{|c|c|c|c|c|c|c|}
\hline \multirow[b]{2}{*}{ Bond } & \multirow{2}{*}{$\begin{array}{l}\text { Phenanth. } \\
R^{\mathrm{e}}\end{array}$} & \multicolumn{2}{|l|}{$\mathrm{C} 4$} & \multirow[b]{2}{*}{ Linker } & \multirow[b]{2}{*}{$\overline{R^{\mathrm{e}}}$} & \multirow[b]{2}{*}{$\Delta$} \\
\hline & & $\overline{R^{\mathrm{e}}}$ & $\Delta$ & & & \\
\hline $\mathrm{a}$ & 1.411 & 1.419 & 0.003 & j (A3-C4) & 1.449 & 0.001 \\
\hline $\mathrm{b}$ & 1.385 & 1.381 & 0.002 & k (A6-C4) & 1.436 & 0.005 \\
\hline$b^{\prime}$ & 1.385 & 1.408 & 0.001 & 1 (C4-C4) & 1.442 & 0.001 \\
\hline $\mathrm{c}$ & 1.416 & 1.421 & 0.003 & & & \\
\hline$c^{\prime}$ & 1.414 & 1.425 & 0.001 & & & \\
\hline d & 1.410 & 1.412 & 0.001 & & & \\
\hline $\mathrm{e}$ & 1.442 & 1.434 & 0.001 & & & \\
\hline $\mathrm{e}^{\prime}$ & 1.446 & 1.432 & 0.001 & & & \\
\hline $\mathrm{f}$ & 1.365 & 1.370 & 0.001 & & & \\
\hline
\end{tabular}

Zahedi [10] by means of the ab initio B3LYP/6-31G(d) approach. The agreement is satisfactory, with the exception of the "bridge bonds" (of the symbols $\mathrm{d}, \mathrm{h}$, and $\mathrm{j}$ ), for which the HSSH values are smaller by ca. $0.02 \AA$ (similar trends are found also in acenes, see "Acenes").

The molecular values of HOMA and its components for phenacenes are presented in Table 12. It seems that these quantities converge to some limiting values in the case of the polyphenacene polymer.

\section{Rylenes}

In Fig. 7, we present the first four members of the family of rylenes. As in the case of the family of phenacenes,

Table 9 C-modules. $\mathrm{R}_{\mathrm{av}}$, EN, GEO, and HOMA values

\begin{tabular}{lllll}
\hline Module & $\mathrm{R}_{\mathrm{av}}(\AA)$ & EN & GEO & HOMA \\
\hline Phenanth. & 1.408 & 0.107 & 0.129 & 0.764 \\
C4 & 1.413 & 0.158 & 0.093 & 0.749 \\
\hline
\end{tabular}


Table 10 "Superbenzene" (9) and K-region PAHs (2). Molecular values of $\mathrm{R}_{\mathrm{av}}$, EN, GEO, and HOMA

\begin{tabular}{lllll}
\hline Molecule & $\mathrm{R}_{\mathrm{av}}(\AA)$ & EN & GEO & HOMA \\
\hline $\mathbf{9}$ & 1.416 & 0.199 & 0.090 & 0.711 \\
$\mathbf{1 7}$ & 1.416 & 0.206 & 0.091 & 0.703 \\
$\mathbf{1 8}$ & 1.417 & 0.213 & 0.091 & 0.696 \\
$\mathbf{1 9}$ & 1.417 & 0.213 & 0.092 & 0.695 \\
$\mathbf{2 0}$ & 1.418 & 0.225 & 0.113 & 0.662
\end{tabular}

Table 11 Phenacenes. HSSH equilibrium C-C bond lengths (in $\AA$ )

\begin{tabular}{|c|c|c|c|c|c|}
\hline \multirow[b]{2}{*}{ Bond } & \multicolumn{5}{|c|}{ Molecule } \\
\hline & 21 & 22 & 23 & 24 & 25 \\
\hline $\mathrm{a}$ & 1.411 & 1.415 & 1.413 & 1.414 & 1.414 \\
\hline $\mathrm{b}$ & 1.385 & 1.382 & 1.383 & 1.382 & 1.383 \\
\hline$b^{\prime}$ & 1.385 & 1.382 & 1.383 & 1.383 & 1.383 \\
\hline $\mathrm{c}$ & 1.416 & 1.419 & 1.418 & 1.418 & 1.418 \\
\hline$c^{\prime}$ & 1.414 & 1.418 & 1.416 & 1.417 & 1.417 \\
\hline $\mathrm{d}$ & 1.410 & 1.411 & 1.411 & 1.411 & 1.411 \\
\hline $\mathrm{e}$ & 1.442 & 1.435 & 1.438 & 1.437 & 1.437 \\
\hline$e^{\prime}$ & 1.446 & 1.439 & 1.442 & 1.441 & 1.441 \\
\hline $\mathrm{f}$ & 1.365 & 1.370 & 1.368 & 1.369 & 1.368 \\
\hline f' & - & - & 1.376 & 1.374 & 1.375 \\
\hline g & - & 1.433 & 1.436 & 1.435 & 1.435 \\
\hline g' & - & - & 1.426 & 1.428 & 1.427 \\
\hline h & - & 1.400 & 1.404 & 1.403 & 1.403 \\
\hline $\mathrm{i}$ & - & - & 1.431 & 1.434 & 1.433 \\
\hline i’ & - & - & - & 1.429 & 1.428 \\
\hline $\mathrm{j}$ & - & - & - & 1.407 & 1.406 \\
\hline k & - & - & - & - & 1.431 \\
\hline k' & - & - & - & - & 1.437 \\
\hline 1 & - & - & - & - & 1.372 \\
\hline
\end{tabular}

Fig. 6 Phenacenes. Hückel graphs, with symbols of symmetry nonequivalent bonds
Table 12 Phenacenes. Molecular values of $\mathrm{R}_{\mathrm{av}}$, EN, GEO, and HOMA

\begin{tabular}{lllll}
\hline Molecule & $\mathrm{R}_{\mathrm{av}}(\AA)$ & EN & GEO & HOMA \\
\hline $\mathbf{2 1}$ & 1.408 & 0.107 & 0.129 & 0.764 \\
$\mathbf{2 2}$ & 1.410 & 0.124 & 0.133 & 0.743 \\
$\mathbf{2 3}$ & 1.411 & 0.135 & 0.136 & 0.729 \\
$\mathbf{2 4}$ & 1.411 & 0.142 & 0.136 & 0.722 \\
$\mathbf{2 5}$ & 1.412 & 0.147 & 0.137 & 0.716 \\
\hline
\end{tabular}

with the growing size rylenes approach a limit of a $\pi$ electron polymer. However, in difference to phenacenes, rylenes display modular architecture, based on the ten $\mathrm{C}$ atom, ten $\pi$-electron B2- and B4-modules (see Fig. 1). They may be considered as analogs of naphthalene (see Fig. 8), a "honorary" B0-module. The bond lengths for the B2- and B4-modules, together with the lengths of the corresponding BB-linkers, are presented in Table 13.

The values of HOMA and its components for the Bmodules are presented in Table 14, and the molecular values of these parameters calculated for rylenes appear in Table 15.

\section{Acenes}

The first six members of the family of acenes are shown in Fig. 8. Acenes are similar to phenacenes of "Phenacenes." They also, with the growing size, approach a limit of a $\pi$ electron polymer. The bond lengths of acenes are presented in Table 16: here also we see no clue to identify transferable modules despite some regularities in bond lengths.

We compared our HSSH equilibrium C-C bond lengths in acenes with those calculated by Firouzi and Zahedi [10] by means of the ab initio B3LYP/6-31G(d) approach. As in the<smiles>C1CCC2C(C1)CCCC1CCCCC12</smiles>

21

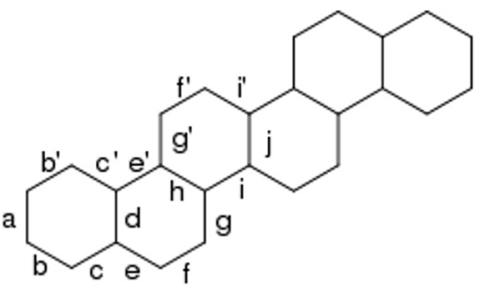

24

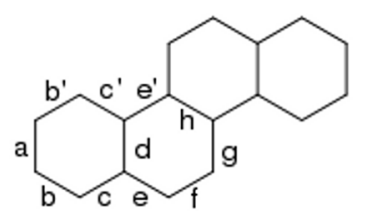

22

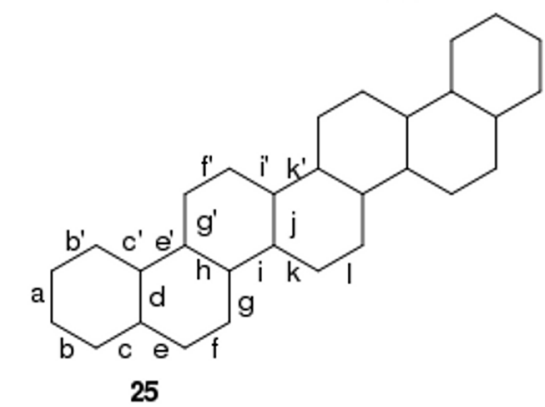


Fig. 7 Rylenes. Hückel graphs, with symbols of symmetry nonequivalent $\mathrm{B}$-modules and BB-linkers
B 2<smiles></smiles>

26
B2

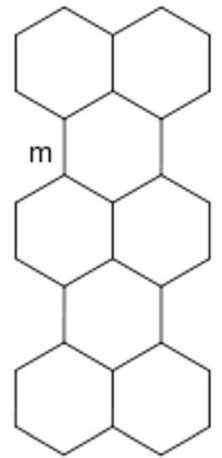

27<smiles>[R5]c1ccc(C(=O)c2ccccc2)cc1</smiles>

28
B2<smiles>C1CC2CCCC3C4CCC5CCCC(C(C1)C23)C54</smiles>

B4

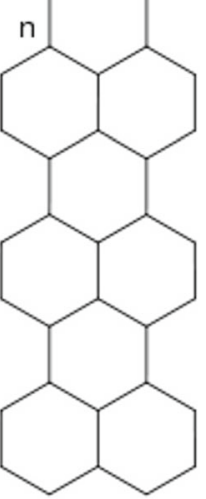

29
Fig. 8 Acenes. Hückel graphs, with symbols of symmetry nonequivalent bonds<smiles>C1CCC2CCCCC2C1</smiles>

Naphthalene<smiles>C1CCC2CC3CCCCC3CC2C1</smiles>

30<smiles>CC12CCCCC1CC1CC3CCCCC3CC12C</smiles>

31<smiles>CC12CCCCC1CC1CC3CC4CCCCC4CC3CC12C</smiles>

32<smiles>C1CCC2CC3CC(=C4CC5CC6CCCCC6CC5CC4C3)CC2C1</smiles><smiles>CC1=C2CC3CC4CC5CCCCC5CC4CC3CC2CC2CC3CCCCC3CC12</smiles>

34

Table 13 B-modules and BB-linkers. HSSH equilibrium C-C bond lengths (in $\AA$ )

\begin{tabular}{|c|c|c|c|c|c|c|c|c|}
\hline \multicolumn{2}{|c|}{ Naphth. } & \multicolumn{2}{|l|}{ B2 } & \multicolumn{2}{|l|}{ B4 } & \multirow[b]{2}{*}{ Linker } & \multirow[b]{2}{*}{$\overline{R^{\mathrm{e}}}$} & \multirow[b]{2}{*}{$\Delta$} \\
\hline Bond & $R^{\mathrm{e}}$ & $\overline{R^{\mathrm{e}}}$ & $\Delta$ & $\overline{R^{\mathrm{e}}}$ & $\Delta$ & & & \\
\hline $\mathrm{a}$ & 1.421 & 1.414 & 0.001 & 1.405 & 0.002 & m' (B2-B2) & 1.461 & - \\
\hline $\mathrm{b}$ & 1.377 & 1.381 & 0.001 & - & - & m (B2-B4) & 1.458 & 0.001 \\
\hline$b^{\prime}$ & - & 1.390 & 0.002 & 1.395 & 0.002 & n (B4-B4) & 1.454 & 0.001 \\
\hline $\mathrm{c}$ & 1.425 & 1.424 & 0.000 & - & - & & & \\
\hline$c^{\prime}$ & - & 1.427 & 0.000 & 1.426 & 0.000 & & & \\
\hline d & 1.414 & 1.415 & 0.000 & 1.417 & 0.001 & & & \\
\hline
\end{tabular}


Table 14 B-modules. $\mathrm{R}_{\mathrm{av}}$, EN, GEO, and HOMA values

\begin{tabular}{lllll}
\hline Module & $\mathrm{R}_{\mathrm{av}}(\AA)$ & EN & GEO & HOMA \\
\hline Naphth. & 1.406 & 0.081 & 0.125 & 0.794 \\
B2 & 1.408 & 0.102 & 0.081 & 0.817 \\
B4 & 1.410 & 0.126 & 0.045 & 0.829 \\
\hline
\end{tabular}

Table 15 Rylenes. Molecular values of $\mathrm{R}_{\mathrm{av}}$, EN, GEO, and HOMA

\begin{tabular}{lllll}
\hline Molecule & $\mathrm{R}_{\mathrm{av}}(\AA)$ & EN & GEO & HOMA \\
\hline $\mathbf{2 6}$ & 1.408 & 0.107 & 0.129 & 0.764 \\
$\mathbf{2 7}$ & 1.410 & 0.124 & 0.133 & 0.743 \\
$\mathbf{2 8}$ & 1.411 & 0.135 & 0.136 & 0.729 \\
$\mathbf{2 9}$ & 1.411 & 0.142 & 0.136 & 0.722 \\
\hline
\end{tabular}

Table 16 Acenes. HSSH equilibrium C-C bond lengths (in $\AA$ ). For benzene $R_{\mathrm{a}}^{\mathrm{e}}=1.397 \AA$

\begin{tabular}{|c|c|c|c|c|c|c|}
\hline \multirow[b]{2}{*}{ Bond } & \multicolumn{6}{|l|}{ Molecule } \\
\hline & Naphth. & 30 & 31 & 32 & 33 & 34 \\
\hline $\mathrm{a}$ & 1.421 & 1.431 & 1.435 & 1.438 & 1.439 & 1.440 \\
\hline $\mathrm{b}$ & 1.377 & 1.370 & 1.367 & 1.366 & 1.365 & 1.364 \\
\hline $\mathrm{c}$ & 1.425 & 1.435 & 1.440 & 1.442 & 1.443 & 1.444 \\
\hline $\mathrm{d}$ & 1.414 & 1.426 & 1.433 & 1.437 & 1.439 & 1.441 \\
\hline $\mathrm{e}$ & - & 1.404 & 1.395 & 1.390 & 1.388 & 1.387 \\
\hline$f$ & - & - & 1.416 & 1.422 & 1.425 & 1.427 \\
\hline $\mathrm{g}$ & - & - & 1.432 & 1.437 & 1.439 & 1.441 \\
\hline h & - & - & - & 1.406 & 1.401 & 1.398 \\
\hline i & - & - & - & - & 1.413 & 1.416 \\
\hline j & - & - & - & - & 1.439 & 1.441 \\
\hline $\mathrm{k}$ & - & - & - & - & - & 1.408 \\
\hline
\end{tabular}

Table 17 Acenes. Molecular values of $\mathrm{R}_{\mathrm{av}}$, EN, GEO, and HOMA

\begin{tabular}{lllll}
\hline Molecule & $\mathrm{R}_{\mathrm{av}}(\AA)$ & EN & GEO & HOMA \\
\hline Benzene & 1.397 & 0.021 & 0.000 & 0.979 \\
Naphth. & 1.406 & 0.081 & 0.125 & 0.794 \\
30 & 1.409 & 0.118 & 0.168 & 0.714 \\
$\mathbf{3 1}$ & 1.411 & 0.141 & 0.181 & 0.678 \\
$\mathbf{3 2}$ & 1.413 & 0.156 & 0.183 & 0.661 \\
$\mathbf{3 3}$ & 1.413 & 0.166 & 0.179 & 0.655 \\
$\mathbf{3 4}$ & 1.414 & 0.174 & 0.173 & 0.653 \\
\hline
\end{tabular}

case of the phenacenes, see "Phenacenes," the agreement is satisfactory, with the exception of the "bridge bonds" (of the symbols $\mathrm{d}, \mathrm{g}$, and $\mathrm{j}$ ), for which the HSSH values are smaller by ca. $0.02 \AA$.

The molecular values of HOMA and its components for acenes are presented in Table 17. As seen, for long acenes the HOMA values drop below the value for graphene, equal to 0.666 (a high chemical reactivity of long acenes is to be noted).

\section{Concluding remarks}

Our HSSH study of the equilibrium C-C bond lengths corresponding to the PAHs considered in the review paper by Riegel and Müllen [3] reveals that some classes of PAHs (clarenes of "Clarenes," K-region PAHs of "K-Region PAHs," and rylenes of "Rylenes") can be described by employing the modular architecture concept. The classes of clarenes and K-region PAHs may be expanded to accommodate PAHs of arbitrary sizes, thus, in a sense, approaching a limit of the graphene lattice contained within a boundary. That boundary, or a perimeter, is built of some An-modules ( $\mathrm{n}=2,3,4,5)$, eventually plus the C4-modules in the case of the K-region PAHs. The interior, corresponding to the "bulk" graphene, is made solely of the A6-modules.

Our present HSSH study of PAHs geometries (see our analysis in "On the path from clarenes to graphene") provides a suggestive indication that the interiors of PAHs, with their growing sizes, converge to the infinite model of graphene, with indistinguishable "full" and "empty" hexagonal rings. In a future paper, we are going to pursue this problem by performing HSSH calculations for very big clarenes and K-region PAHs (with hundreds to thousands carbon atoms).

Author contribution L. Z Stolarczyk (the concept of the paper, the computer code, the analysis of the results, writing the first draft of the paper). J. H. Kwapisz (writing the input data, performing the calculations, the analysis of the results, making the tables and figures, preparing the final form of the paper).

Availability of data and materials No additional data is of relevance.

\section{Declarations}

Consent for publication The co-author (J. H. Kwapisz) is aware and approve of the submission.

Conflict of interest The authors declare no competing interests.

Open Access This article is licensed under a Creative Commons Attribution 4.0 International License, which permits use, sharing, 
adaptation, distribution and reproduction in any medium or format, as long as you give appropriate credit to the original author(s) and the source, provide a link to the Creative Commons licence, and indicate if changes were made. The images or other third party material in this article are included in the article's Creative Commons licence, unless indicated otherwise in a credit line to the material. If material is not included in the article's Creative Commons licence and your intended use is not permitted by statutory regulation or exceeds the permitted use, you will need to obtain permission directly from the copyright holder. To view a copy of this licence, visit http://creativecommons. org/licenses/by/4.0/.

\section{References}

1. Hopf H (2000) Classics in hydrocarbon chemistry. Wiley-VCH, Weinheim

2. Kubozono Y (2019) Physics and chemistry of carbon-based materials. Springer Nature, Singapore

3. Riegel RR, Müllen K (2010) Forever young: polycyclic aromatic hydrocarbons as model cases for structural and optical studies. J Phys Org Chem 23:315-325

4. Stolarczyk LZ, Krygowski TM (2021) Augmented Hückel molecular orbital model of $\pi$-electron systems: from topology to metric. I. General theory. J Phys Org Chem 34(1-15):e4154. Paper I

5. Stolarczyk LZ, Krygowski TM (2021) Augmented Hückel molecular orbital model of $\pi$-electron systems: from topology to metric. II. Hydrocarbon and carbon molecules. J Phys Org Chem 34(1-13):e4153. Paper II

6. Kiralj R, Ferreira MMC (2002) Predicting Bond Lengths in Planar Benzenoid Polycyclic Aromatic Hydrocarbons: A Chemometric Approach. J Chem Inf Comput Sci 42:508-523

7. Brock CP, Dunitz JD, Hirshfeld FL (1991) Transferability of deformation densities among related molecules: Atomic multipole parameters from perylene for improved estimation of molecular vibrations in naphthalene and anthracene. Acta Crystallogr Sect B 47:789-797

8. Kiralj R, Kojić-Prodić B, Nikolić S, Trinajstić N (1998) Bond lengths and bond orders in benzenoid hydrocarbons and related systems: a comparison of valence bond and molecular orbital treatments. J Mol Struct THEOCHEM 427:25-37

9. Kadantsev ES, Stott MJ, Rubio A (2006) Electronic structure and excitations in oligoacenes from ab initio calculations. J Chem Phys 124(1-11):134901

10. Firouzi R, Zahedi M (2008) Polyacenes electronic properties and their dependence on molecular size. J Mol Struct THEOCHEM 862:7-15

11. Clar E (1972) The aromatic sextet. Wiley, New York

12. Solà M (2013) Forty years of Clar's aromatic $\pi$-sextet rule. Front Chem Lausanne Switz 1(1-8):22
13. Yamaguchi Y, Osamura Y, Goddard JD, Schaefer III HF (1994) A new dimension to quantum chemistry analytic derivative methods in ab initio molecular electronic structure theory. Oxford University Press, Oxford

14. Coulson CA, Rushbrooke GS (1940) Note on the method of molecular orbitals. Proc Camb Philos Soc 36:193-200

15. Kruszewski J, Krygowski TM (1972) Definition of aromaticity basing on the harmonic oscillator model. Tetrahedron Lett 13:3839-3842

16. Krygowski TM (1993) Crystallographic studies of inter- and intramolecular interations reflected in aromatic character of $\pi$ electron systems. J Chem Inf Comput Sci 33:70-78

17. Krygowski TM, Cyrański M (1996) Separation of the energetic and geometric contributions to the aromaticity of $\pi$-electron carbocyclics. Tetrahedron 52:1713-1722

18. Cyrański MK, Krygowski TM (1999) Two sources of the decrease of aromaticity: Bond length alternation and bond elongation. Part I. An analysis based on benzene ring deformations. Tetrahedron 55:6205-6210

19. Trinajstić N (1992) Chemical Graph Theory, 2nd edn. CRC Press, Boca Raton FL

20. Scott LT (2015) Chemistry at the interior atoms of polycyclic aromatic hydrocarbons. Chem Soc Rev 44:6464-6471

21. Tyutyulkov N, Madjarova G, Dietz F, Müllen K (1998) Is 2-D graphite an ultimate large hydrocarbon? 1. Energy spectra of giant polycyclic aromatic hydrocarbons. J Phys Chem B 102:1018310189

22. Dietz F, Tyutyulkov N, Madjarova G, Müllen K (2000) Is 2-D graphite an ultimate large hydrocarbon? II. Structure and energy spectra of polycyclic aromatic hydrocarbons with defects. J Phys Chem B 104:1746-1761

23. Tyutyulkov N, Müllen K, Baumgarten M, Ivanova A, Tadjer A (2003) Is 2-D graphite an ultimate large hydrocarbon? III. Structure and energy spectra of large polybenzenoid hydrocarbons with different edge structures. Synth Met 139:99-107

24. Staykov A, Gehrgel L, Dietz F, Tyutyulkov N (2003) Is 2D graphite an ultimate large hydrocarbon? IV. Structure and energy spectra of polycyclic aromatic hydrocarbons with different symmetry. Z Naturforsch B J Chem Sci 58b:965-970

25. Dickens TK, Mallion RB (2018) Topological ring-currents and Clar sextets in fully benzenoid hydrocarbons I. Structures with fewer than 18 Rings. J Phys Chem A 122:8858-8864

26. Dickens TK, Mallion RB (2018) Topological ring-currents and Clar sextets in fully benzenoid hydrocarbons. II. Large structures containing more than 18 rings. J Phys Chem A 122:8865-8873

27. Iyer VS, Wehmeier M, Brand JD, Keegstra MA, Müllen K (1997) From hexa-peri-hexabenzocoronene to superacenes. Angew Chem Int Ed Engl 36:1604-1607

Publisher's note Springer Nature remains neutral with regard to jurisdictional claims in published maps and institutional affiliations. 